\title{
Remote Information and Power Transfer for Multi Relay-Assisted Agreeable Communication
}

\author{
G. Harish Kumar, S. Srikanth
}

\begin{abstract}
In this paper, we studied about Simultaneous wireless information and power transfer (SWIPT) in multi relay-helped two-jump hand-off framework, where different hand-off hubs all the while help the transmission from source to goal utilizing the idea of dispersed space-time coding. Each hand-off applies control part convention to arrange the got flag vitality for data deciphering and vitality gathering. The streamlining issues of intensity part proportions at the transfers are planned for both decipher and-forward $(D F)$ and intensify and-forward (AF) handing-off conventions. Productive calculations are proposed to discover the ideal arrangements. Recreations check the viability of the proposed plans.
\end{abstract}

Index Terms -Simultaneous wireless information and power transfer (SWIPT), energy harvesting, cooperative communication, and resource allocation.

\section{INTRODUCTION}

ENERGY reaping has risen as a promising methodology to delay lifetime of vitality obliged remote systems. Be that as it may, the customary vitality collecting advances depend on common vitality sources (like sunlight based, wind, vibration and so on.), which can't be controlled and are not constantly accessible. As of late, radio-frequency (RF) signals transmitted by encompassing transmitters turns into a suitable new hotspot for remote vitality gathering. Since RF signals convey vitality and also data in the meantime, Simultaneous wireless information and power transfer (SWIPT) empowers productive asset assignment at handset structures and along these lines has drawn a huge consideration in remote interchanges. Two practical schemes for wireless information and power transfer were proposed in [1] and [2], namely "time switching" where the receiver switches between decoding information and harvesting energy, and "power splitting" where the receiver splits the signal power into two parts for decoding information and harvesting energy. The authors in [3] and [4] investigated capacity-energy tradeoff for time switching protocol and the power splitting protocol, respectively. SWIPT has been studied for various aspects, such as energy efficiency [5] and physical-layer security [6]-[8].

In helpful or sensor arranges the middle of the road hand-off hubs frequently have less battery stockpiling and require outer charging to stay dynamic. Along these lines vitality gathering is more imperative for transfer or sensor

Revised Manuscript Received on 14 August, 2019.

G. Harish Kumar, Asst. Professor, Dept. of ECE, Malla Reddy Engineering College for Women(Autonomous), Secunderabad-TS harish.gopadi@gmail.com

S.Srikanth, Asst. Professor, Dept. of ECE, Malla Reddy Engineering College for Women(Autonomous), Secunderabad-TS srimrecw@gmail.com hubs. A bunch of works contemplated SWIPT in helpful hand-off frameworks. For example, the blackout likelihood and the ergodic limit with regards to time exchanging and control part conventions were inferred in [9], where the hand-off harvests a small amount of vitality from the source, and the hand-off utilizes the reaped vitality to forward the source's data to the goal. In [10], the creators examined remote data and power move in helpful systems where the arbitrarily found transfers help one source-goal match; furthermore, blackout likelihood and decent variety gain were described by stochastic geometry. The creators in [11] contemplated the blackout execution of intensity techniques at a vitality collecting hand-off for different source-goal sets. The ideal time switching proportion was explored in full-duplex transferring framework under various correspondence modes in [12]. Power part for obstruction transfer channels utilizing amusement hypothesis was proposed in [13]. The creators in [14] considered secure transfer beam forming structure with SWIPT in two-bounce non-regenerative transfer frameworks. Effective imperfect calculations for SWIPT in different information numerous yield orthogonal frequency division multiplexing (MIMO-OFDM) non-regenerative transferring were proposed in [15]. Conventions and streamlining for SWIPT based OFDM transferring were examined in [16] Self-vitality reusing for remote controlled transfer was contemplated in [17]. In [18], the creators considered the time exchanging convention in multi-transfer system, and joint vitality reaping and beam forming plans were proposed.

In perspective of the related takes a shot at SWIPT in hand-off frameworks, it is discovered that the vast majority of these works accept that just a single hand-off helps the source-goal transmission. Despite the fact that the work [10] considered numerous transfers, the creators concentrated on blackout examination with settled power part coefficients. As the RF signals transmitted by the source can be gotten by all transfers, the different transfers can at the same time facilitate the got vitality and data utilizing similar signs from the source. Along these lines, without additional source control, the framework execution can be significantly enhanced by using the capability of spatial assorted variety of the disseminated transfer hubs. This rouses our contemplate.

In this paper, we consider SWIPT in two-bounce handing-off frameworks, where the numerous transfers help the transmission from the source to goal. The source hub is with settled vitality supply, and the hand-off hubs have no vitality or are not willing to consume their very own vitality to encourage the source. Accepting control part connected at the transfer collectors, the transfers split a bit of the got vitality as the wellspring of capacity to forward the signs of 
the source by utilizing dispersed space-time codes [19]- [22]. Both Decode and-forward (DF) and Amplify and-forward (AF) handing-off conventions are considered. We will likely boost the conclusion to-end rate by enhancing the transfers' capacity part proportions. We locate the ideal arrangements by effective calculations. The rest of the letter is composed as pursues: Section II portrays the framework show. Issue definitions and proposed calculations are itemized in Section III. Recreations results are given in Section IV. At long last we finish up the letter in Area V.

\section{SYSTEM MODEL}

We consider a two-hop relay network model as shown in Fig. 1 , where multiple relays assist the transmission from the source $\mathrm{S}$ to the destination $\mathrm{D}$. All nodes are equipped with a single Omni directional antenna. The relay set is denoted as $\mathrm{R}=\{1, \ldots, \mathrm{K}\}$. We assume that the direct link between $\mathrm{S}$ and $\mathrm{D}$ is blocked due to the shielding effect caused by obstacles, which is a well-known Type-II relay model in 3GPP LTE-A. Such that the transmit signals from $\mathrm{S}$ need to be forwarded by the relay set $R$. Denote $h_{i}$ as the channel gain between $S$ and relay $i$, and $g_{i}$ as the channel gain between relay $i$ and $D$. Moreover, we assume that the additive white Gaussian noises (AWGN) at all nodes are independent circular symmetric complex Gaussian random variables, each having zero mean and unit variance. The channel fading is modeled by large-scale path loss and small scale Rayleigh fading. The transmission from the source to destination divided into consecutive frames. It is assumed that the channel fading remains unchanged within each transmission frame but varies from one frame to another. We also assume that perfect channel state information (CSI) is available at all nodes.

Each transfer hub has the vitality reaping capacity to gather vitality from the got flags by power part. The source $S$ is with a settled vitality supply, while each hand-off has no vitality (or on the other hand isn't willing to consume its own vitality) to encourage the source, be that as it may, they can forward the source's data by utilizing the vitality gathered from the source. All the more explicitly, each hand-off I parts a bit of the got flag vitality $\alpha$ f for data unraveling, and the rest section $1-\alpha i$ for vitality gathering. The collected vitality at each hand-off is utilized as the transmit control for sending the source's data. The hand-off hubs work on half-duplex mode with the goal that the two-bounce transmission is isolated into two stages. In the main stage, $\mathrm{S}$ communicates the signs to the transfers. In the second stage, all transfers shape a disseminated MIMO framework and all the while forward the signs to the goal by utilizing appropriated space-time codes.

\section{OPTIMIZATION FRAMEWORK}

In this section, we detail the issue plans and ideal answers for both DF and AF, separately.

A. Unravel and-Forward Relaying

Give $\mathrm{P}$ a chance to signify the transmit intensity of the source. For each hand-off hub I, they got flag vitality is Phi . By power part with the proportion $\alpha \mathrm{i}$, a segment of $\mathrm{Ph}_{\mathrm{i}} \alpha \mathrm{i}$ is utilized to data deciphering and the rest $\operatorname{Phi}\left(1-\alpha_{\mathrm{i}}\right)$ is contribution to vitality collector as the hand-off power. For multi-transfer helped DF convention, the flag to-commotion proportion (SNR) of the first and second jumps are given by [19], [20], separately

$$
\mathrm{SNR}_{\mathrm{DF}, 1}=\min _{\mathrm{i} \in \mathrm{R}} \mathrm{Ph}_{\mathrm{i}} \alpha_{\mathrm{i}}
$$

and

$$
\mathrm{SNR}_{\mathrm{DF}, 2}=\underset{\mathrm{i} \in \mathrm{R}}{\zeta \mathrm{Ph}_{\mathrm{i}}(1-\alpha \mathrm{i}) \mathrm{g}_{\mathrm{i}}}
$$

where $\zeta$ is the vitality transformation proficiency. It is prominent that in conventional DF conspire without power part (or $\alpha i=1$ ), the source transmit rate is restricted by the most exceedingly terrible channel gain hey among the hand-off set $\mathrm{R}$ in the principal bounce. Be that as it may, by considering control part at transfers, the source transmit rate is constrained by the base got SNR among the transfer set, which altogether muddles the issue and makes this examine nontrivial. To guarantee the dependable recuperation of the transmit motion at each transfer in $\mathrm{R}$, the source transmit rate ought not be higher than $\log 2(1+\mathrm{SNRDF}, 1)$. Also, the source transmit rate ought to not be higher than $\log 2(1+$ SNRDF,2) to guarantee dependable flag interpreting at the goal. Along these lines, the conclusion to-end rate ought to be [19], [20].

$\mathrm{RDF} \leq \Sigma 1 / 2 \min \log _{2}\left(1+\mathrm{SNR}_{\mathrm{DF},}, 1\right), \quad \log _{2}\left(1+\mathrm{SNR}_{\mathrm{DF}, 2}\right)$ (3)

Here the factor $1 / 2$ is because of the helpful transmission taking two schedule openings. We will likely boost the conclusion to-end rate by upgrading the power part proportions of the transfers. The issue can be planned as

P1 : $\operatorname{maxRDF}$

s.t. $0 \leq \alpha_{\mathrm{i}} \leq 1, \forall \mathrm{i} \in \mathrm{R}$.

Note that in (3) both RDF $\leq 1 / 2 \log 2(1+\mathrm{SNRDF}, 1)$ and RDF $\leq 1 / 2 \log 2(1+\mathrm{SNRDF}, 2)$ are curved sets, as is their convergence. Also, the imperative (4b) is undertaking. In this way the issue $\mathrm{P} 1$ is a raised issue. We can locate the ideal arrangement with shut shape via painstakingly investigating the structure of the rate articulation. In the accompanying we detail the determination. Initially, for the DF case, the ideal arrangement must occur at SNRDF,1 = SNRDF,2, which infers that the condition

$\min \mathrm{Ph}_{\mathrm{i}} \alpha_{\mathrm{i}}=. \zeta \mathrm{Ph}_{\mathrm{i}}\left(1-\alpha_{\mathrm{i}}\right) \mathrm{g}_{\mathrm{i}}$

$i \in R \quad i \in R$

Ought to be constantly fulfilled. To this end, we find that the following condition must hold for every $\mathrm{I} \in \mathrm{R}$ at the ideal:

$$
\begin{aligned}
& \mathrm{Ph}_{\mathrm{i}} \alpha_{\mathrm{i}}=\zeta \mathrm{Ph}_{\mathrm{i}}\left(1-\alpha_{\mathrm{i}}\right) \mathrm{g}_{\mathrm{i}} 3 / 4 \mathrm{SNR}_{\mathrm{eff}}, \forall \mathrm{i} \in \mathrm{R} \\
& i \in R
\end{aligned}
$$

Where $\mathrm{SNR}_{\mathrm{eff}}$ is the powerful SNR and is a consistent. This implies that they got SNR at all transfers ought to be the equivalent at the ideal. At that point we have

$$
\text { SNReff }=\Sigma \zeta \mathrm{Ph}_{\mathrm{i}}\left(1-\left(\mathrm{SNReff} / \mathrm{Ph}_{\mathrm{i}}\right) \mathrm{g}_{\mathrm{i}}\right.
$$

$$
i \epsilon R
$$

Combining the constraint (4b), we get $=\min (\Sigma \zeta$ Phi gi $/(1+\Sigma \zeta$ Phi gi $) \quad(8)$

At long last, the ideal power part proportion at each transfer can be gotten as

$$
\mathrm{SNR}_{\mathrm{eff}}=\min \left(\Sigma \zeta \mathrm{Phi}_{\mathrm{i}} /\left(1+\Sigma \zeta \mathrm{Phi} \mathrm{g}_{\mathrm{i}}\right)\right.
$$

\section{.} . 
B. Enhance and-Forward Relaying

For the AF case, the rate articulation can be effortlessly gotten as [19], [20]

$\mathrm{R}_{\mathrm{AF}}=\Sigma 1 / 2 \min \log _{2} \Sigma\left(1+\right.$ Phiai $+\zeta$ Phi $\left.(1-\alpha \mathrm{i}) \mathrm{g}_{\mathrm{i}}\right)$ (10)

Like the DF case, the issue plan of the AF case can be communicated as

P2:maxRAF

s.t. $0 \leq \alpha \mathrm{i} \leq 1, \forall \mathrm{i} \in \mathrm{R}$

$\mathrm{P} 2$ is a non convex issue since the rate articulation RAF is not inward. In the accompanying, we locate its ideal arrangement in an productive way.

To begin with, it is seen that the summation term in (10) is decomposable since $\left\{\alpha_{i}\right\}$ are free one another. Consolidating the certainty that boosting $\log 2(1+x)$ is comparable to augmenting $\mathrm{x}$, we can deteriorate the first issue into $\mathrm{K}$ autonomous subproblems, each comparing to one transfer and having the indistinguishable structure. Each subproblem can be communicated as

$$
\text { s.t. } 0 \leq \alpha_{i} \leq 1 \text {. }
$$

The issue in (12) is still nonconvex. Notwithstanding, it is promptly affirmed that the numerator of (12a) is nonnegative and sunken, while the denominator is raised and positive. In this manner, the issue in (12) is a sunken partial programming issue [23]. By characterizing $\varphi_{1}(\alpha \mathrm{i}) \alpha_{\mathrm{i}}\left(1-\alpha_{\mathrm{i}}\right)$ and $\varphi 2\left(\alpha_{i}\right) P h_{i} \alpha_{i}+\zeta \operatorname{Phi}\left(1-\alpha_{i}\right) g_{i}$, and presenting the new factors $x_{i} \alpha_{i} \varphi 2\left(\alpha_{i}\right)$ furthermore, $y_{i} 1 \varphi 2\left(\alpha_{i}\right)$, the issue in (12) can be changed as

$$
\begin{aligned}
& \operatorname{Max} \zeta P^{2} h^{2} g_{i} y_{i} \varphi_{1}\left(x_{i} / y_{i}\right) \\
& x_{i}, y i \\
& \text { s.t. } \quad y_{i} \varphi_{2}\left(x_{i} / y_{i}\right) \leq 1 \\
& x_{i} \leq y_{i} \\
& x_{i} \geq 0, y_{i}>0 .
\end{aligned}
$$

Presently the issue in (13) is an arched issue and can be fathomed effectively utilizing the inside point technique. Subsequent to acquiring the ideal $\mathrm{x}^{*}{ }_{\mathrm{i}}$ and $\mathrm{y}^{*}{ }_{\mathrm{i}}$, the ideal $\alpha^{*}{ }_{\mathrm{i}}$ can be recouped by letting $\alpha^{*}{ }_{\mathrm{i}}=\mathrm{x}_{\mathrm{i}}^{*} / \mathrm{y}^{*}{ }_{\mathrm{i}}$. Comment 1: Our enhancement structure does not require a brought together controller and the ideal power part proportion $\alpha^{*}{ }_{i}$ can be controlled by individual transfers. In particular, for the DF case, each transfer i communicates its channel picks up $h_{i}$ there and gi, in this manner each hand-off $i$ can realize all channel gains $\{\mathrm{hi}\}$ and $\left\{\mathrm{g}_{\mathrm{i}}\right\}$ and afterward decide $\alpha_{\mathrm{i}}^{*}$ utilizing (9). For AF case, the first issue P2 is decoupled into K subproblems as (12), where each subproblem compares to one transfer for discovering $\alpha^{*}{ }_{i}$ utilizing its very own channel gains $h_{i}$ and $g_{i}$. In this way, in our letter, the ideal arrangements for both DF and AF can be found distributed.

\section{SIMULATION RESULTS}

In this segment, we assess the proposed plans by means of reenactments. We consider a two-dimensional plane where the source what's more, goal are settled at $(0,0)$ and $(10,0)$ $\mathrm{m}$, separately, what's more, the transfer hubs are haphazardly however consistently dispersed in a square with length of $2 \mathrm{~m}$ whose main issue is hanging in the balance between the source and goal. $K=5$ transfers are situated in the square. The main issue of the transfer square is at $d=(5,0)$, i.e., the hand-off locale is at the center of the source and goal. Each blurring is demonstrated as $\mathrm{c} \cdot \mathrm{L}^{-\theta}$, where $\mathrm{c}$ is the Rayleigh blurring factor, $\mathrm{L}$ is transmitter-recipient separation, and $\theta$ is the way misfortune example which is set to be 3 . We set the vitality transformation productivity coefficient $\zeta=60 \%$. We initially think about the customary best transfer determination (BRS) as the execution benchmark: Assuming that the transfer set $\mathrm{R}$ contains just a single hand-off hub, the ideal source power and power part proportion can be gotten utilizing the proposed calculations for both DF and AF; at that point complete a thorough hunt over all transfers also, select a transfer that outcomes in best framework execution. Fig. 2 thinks about rate exhibitions of various plans. It is seen that the proposed DF and AF plans perform

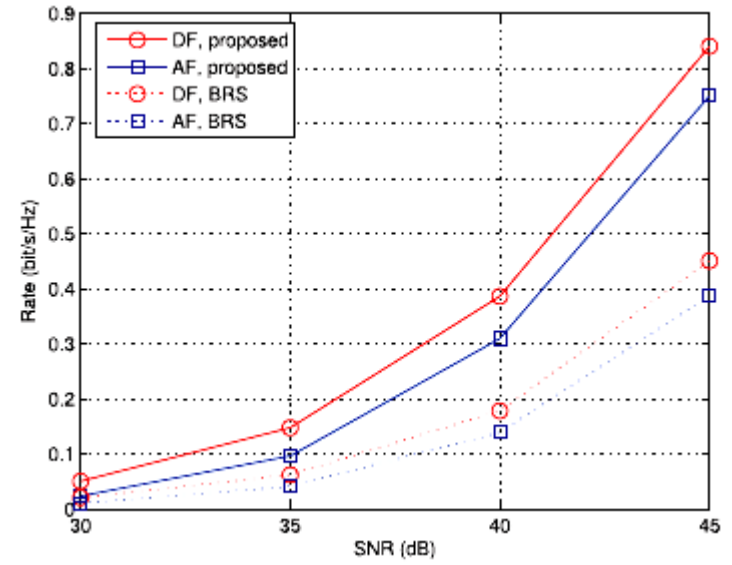

Fig. 2. Performance comparison of different schemes.

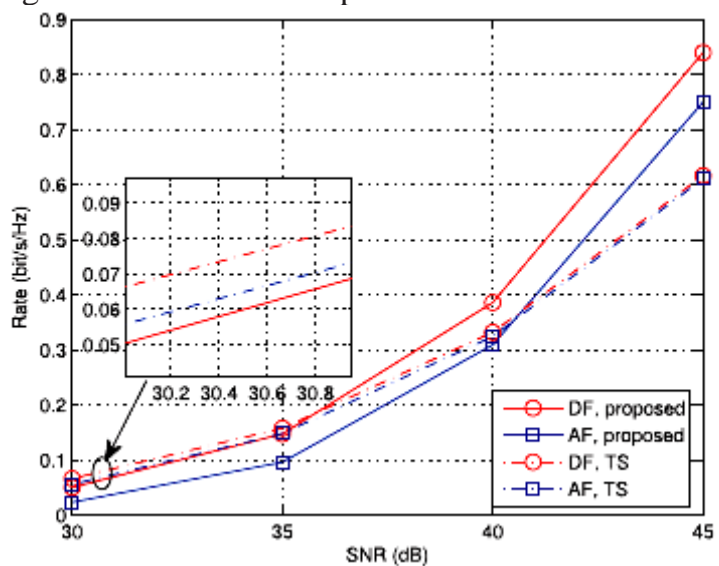

Fig. 3. Performance comparison with time switching protocol.

Nearly, and the DF conspire is somewhat better than the AF conspire. In addition, we likewise see that the proposed plans essentially beat the customary BRS plans, which shows the prevalence of the proposed plans. Note again that the execution gain contrasted with the BRS plans is with no additional source control, since the transfers utilize the equivalent signs of the source. Subsequently the proposed plans are best indeed, even in a vitality effectiveness point of view. We at that point contrast the proposed power part plots and time switching (TS) conspires in [18], where we comprehensively look through the time assignment variable $t$ over the range $[0,1]$ with small interims for finding the ideal $t^{*}$ that makes the rate most extreme. We see that the power part convention outflanks the time exchanging convention in high SNR locale, while the time exchanging convention is better in low SNR area. Our perception is as per the outcomes in [9] where single relay is considered.

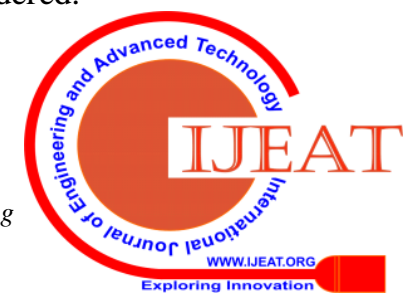




\section{CONCLUSION}

This paper considered concurrent remote data and control move in multi-transfer helped two-jump agreeable correspondence, where various transfer hubs use control part convention to at the same time organize the utilization of the got signals for data deciphering and vitality reaping. Power part proportions at the transfers were enhanced for both DF and AF transferring methodologies. Reproduction results demonstrate that the proposed conspires altogether beat the conventional best transfer determination plans. Besides, we show that control part is better in high SNR district and time exchanging is supported in low SNR area in hand-off systems.

\section{REFERENCES}

1. R. Zhang and C. Ho, "MIMO broadcasting for simultaneous wireless information and power transfer," IEEE Trans. Wireless Commun., vol. 12, no. 5, pp. 1989-2001, May 2013.

2. X. Zhou, R. Zhang, and C. Ho, "Wireless information and power trans- fer: Architecture design and rate-energy tradeoff," IEEE Trans. Commun., vol. 61, no. 11, pp. 4754-4767, Nov. 2013.

3. L. Liu, R. Zhang, and K. C. Chua, "Wireless information transfer with opportunistic energy harvesting," IEEE Trans. Wireless Commun., vol. 12, no. 1, pp. 288-300, Jan. 2013.

4. L. Liu, R. Zhang, and K. C. Chua, "Wireless information and power transfer: A dynamic power splitting approach,” IEEE Trans. Commun., vol. 61, no. 9, pp. 3990-4001, Sep. 2013.

5. D. W. K. Ng, E. S. Lo, and R. Schober, "Wireless information and power transfer: Energy efficiency optimization in OFDMA systems," IEEE Trans. Wireless Commun., vol. 12, no. 12, pp. 6352-6370, Dec. 2013.

6. D. W. K. Ng, E. S. Lo, and R. Schober, "Robust beamforming for secure communication in systems with wireless information and power trans- fer," IEEE Trans. Wireless Commun., vol. 13, no. 8, pp. 4599-4615, Aug. 2014.

7. M. Zhang and Y. Liu, "Energy harvesting for physical-layer security in OFDMA networks,” IEEE Trans. Inf. Forensics Secur., vol. 11, no. 1, pp. 154-162, Jan. 2016.

8. M. Zhang, Y. Liu, and R. Zhang, "Artificial noise aided secrecy information and power transfer in OFDMA systems," IEEE Trans. Wireless Commun., arXiv:1601.01435, 2016, to be published

9. Nasir, X. Zhou, S. Durrani, and R. A. Kennedy, "Relaying protocols for wireless energy harvesting and information processing," IEEE Trans. Wireless Commun., vol. 12, no. 7, pp. 3622-3636, Jul. 2013.

10. Z. Ding, I. Krikidis, B. Sharif, and H. Poor, "Wireless information and power transfer in cooperative networks with spatially random relays," IEEE Trans. Wireless Commun., vol. 13, no. 8, pp. 4440-4453, Aug. 2014.

11. Z. Ding, S. Perlaza, I. Esnaola, and H. Poor, "Power allocation strate- gies in energy harvesting wireless cooperative networks," IEEE Trans. Wireless Commun., vol. 13, no. 2, pp. 846-860, Feb. 2014.

12. C. Zhong, H. Suraweera, G. Zheng, I. Krikidis, and Z. Zhang, "Wireless information and power transfer with full duplex relaying," IEEE Trans. Commun., vol. 62, no. 10, pp. 3447-3461, Oct. 2014.

13. H. Chen, Y. Li, Y. Jiang, Y. Ma, and B. Vucetic, "Distributed power splitting for SWIPT in relay interference channels using game theory," IEEE Trans. Wireless Commun., vol. 14, no. 1, pp. 410-420, Jan. 2015.

14. Q. Li, Q. Zhang, and J. Qin, "Secure relay beamforming for simulta- neous wireless information and power transfer in nonregenerative relay networks," IEEE Trans. Veh. Technol., vol. 63, no. 5, pp. 2462-2467, Jun. 2014.

15. K. Xiong, P. Fan, C. Zhang, and K. Letaief, "Wireless information and energy transfer for two-hop non-regenerative MIMO-OFDM relay networks," IEEE J. Sel. Areas Commun., vol. 33, no. 8, pp. 1595-1611, Aug. 2015.

16. Y. Liu and X. Wang, "Information and energy cooperation in OFDM relaying: Protocols and optimization," IEEE Trans. Veh. Technol., arXiv:1506.06850, 2015, to be published.

17. Y. Zeng and R. Zhang, "Full-duplex wireless-powered relay with selfenergy recycling," IEEE Wireless Commun. Lett., vol. 4, no. 2, pp. 201204, Apr. 2015.

18. S. Gong, L. Duan, and N. Gautam, "Optimal scheduling and beamforming in relay networks with energy harvesting constraints," IEEE Trans. Wireless Commun., 2015, to be published.

19. J. Laneman and G. W. Wornell, "Distributed space-time-coded protocols for exploiting cooperative diversity in wireless networks," IEEE Trans. Inf. Theory, vol. 49, no. 10, pp. 2415-2425, Oct. 2003.
20. J. Laneman, D. Tse, and G. W. Wornell, "Cooperative diversity in wireless networks: Efficient protocols and outage behavior,” IEEE Trans. Inf. Theory, vol. 50, no. 12, pp. 3062-3080, Dec. 2004.

21. Y. Jing and B. Hassibi, "Distributed space-time coding in wireless relay networks," IEEE Trans. Wireless Commun., vol. 5, no. 12, pp. 3524-3536, Dec. 2006.

22. S. Yiu, R. Schober, and L. Lampe, "Distributed space-time block coding,"

23. N. Nithiyanandam, K. Venkatesh, M. Rajesh, Transfer The Levels Of The Monitored Carbon, Nitrogen Gases From The Industries, International Journal of Recent Technology and Engineering, Volume-7 Issue-6S3 April, 2019.

24. Sivanesh Kumar, A., Brittoraj, S., Rajesh, M., Implementation of RFID with internet of things, Journal of Recent Technology and Engineering, Volume-7 Issue-6S3 April, 2019.

25. Rajesh, M., Sairam, R., Big data and health care system using mlearningJournal of Recent Technology and Engineering, Volume-7 Issue-6S3 April, 2019.

26. Rajesh, M., and J. M. Gnanasekar. "Path Observation Based Physical Routing Protocol for Wireless Ad Hoc Networks." Wireless Personal Communications 97.1 (2017): 1267-1289. 